\title{
Melting of aluminium clusters
}

\author{
E. G. Noya and J. P. K. Doye \\ University Chemical Laboratory, Lensfield Road, Cambridge CB2 1EW, United Kingdom \\ F. Calvo \\ Laboratoire de Physique Quantique, IRSAMC, Université Paul Sabatier, \\ 118 Route de Narbonne, F31062 Toulouse Cedex, France
}

(Dated: November 20, 2018)

\begin{abstract}
The melting of Al clusters in the size range $49 \leq N \leq 62$ has been studied using two model interatomic potentials. The results for the two models are significantly different. The glue potential exhibits a smooth relatively featureless heat capacity curve for all sizes except for $N=54$ and $N=55$, sizes at which icosahedral structures are favoured over the polytetrahedral. Gupta heat capacity curves, instead, show a well-defined peak that is indicative of a first-order-like transition. The differences between the two models reflect the different ground-state structures, and neither potential is able to reproduce or explain the size dependence of the melting transition recently observed in experiments.
\end{abstract}

\section{INTRODUCTION}

Cluster thermodynamics has been an active field of research during the last decades. It is now accepted that clusters do not melt at a singular temperature, but, instead, there is a finite range of temperatures over which solid-like and liquid-like isomers coexist $\underline{\underline{1}}$ Nevertheless, in the cases for which a well-defined peak is observed in the heat capacity curves, melting is generally considered as the finite size analogue of a first-order phase transition ${ }^{2}$ Except for some particular cases, 3.4 clusters usually melt at a temperature lower than the bulk melting temperature, and this temperature generally decreases with the cluster size. The appearance of premelting effects, evidenced by a small peak in the heat capacity curve or by a change of slope in the caloric curve before melting, is also fairly common.

Recently, the melting behaviour of positively charged aluminium clusters with 49 to 62 atoms has been measured. $\frac{5}{5}$ Except for $N=49$ and 59 , the heat capacity curves show well-defined peaks between 450 and 650 $\mathrm{K}$. The melting temperature has an irregular variation with the cluster size, suffering a sharp drop at $N=56$, which was attributed to a structural transition. A plot of the latent heat against temperature resulted in peaks at sizes $N=51,57$ and 61 . Moreover, premelting effects were observed $100 \mathrm{~K}$ before melting for $\mathrm{Al}_{51}^{+}$and $\mathrm{Al}_{52}^{+}$. In order to try to understand the origin of the features observed experimentally, a theoretical study of the thermal properties would be valuable.

The existing literature on $\mathrm{Al}$ clusters has mainly focused on the structural and electronic properties. However, in spite of the experimenta ${ }^{6.7}$

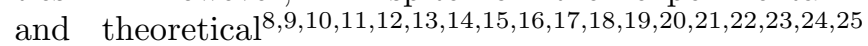
effort, a consensus has not yet been achieved about the structure of many of the experimentally observed magic numbers. Only for large sizes $(N>250)$ the structure of $\mathrm{Al}$ clusters seems to have been rationalized. Martin et al. showed that, above this size, magic numbers were due to geometric shell closings associated with face- centered-cubic octahedra (fcc) $\frac{7}{?}$ and this hypothesis is consistent with results of kinetic simulations $\stackrel{14,15,16}{\text { For }}$ smaller clusters, $a b$ initio calculations have been performed at selected sizes $\stackrel{8,9,11,12,13}{ }$ Only at $N=13$ was agreement on the structure reached, which is thought to be icosahedral 9.11.12.13 The structures at $N=55$ and 147 remain unassigned, as different theoretical calculations found very different structures. In particular, for $N=55$, icosahedral, $\stackrel{11}{\rightleftharpoons}$ decahedral,$\stackrel{13}{=}$ cuboctahedral ${ }^{18}$ and disordered ${ }^{9}$ structures were found most stable, depending on the method used.

The lower computational expense of empirical potentials have allowed more extensive searchs 19.20 .21 .22 .23 .24 .25 However, the results show a strong dependence on the model potential used. The Murrell-Mottram potential ${ }^{26}$ predicts a competition between fcc and icosahedral structures in the size regime $N=2-55$, with strong magic numbers for the 38-atom truncated octahedron and the 54-atom uncentered Mackay icosahedron 18.19 .20 Similar results were obtained with Voter-Chen 27 and Gupta 28 models. Both show special stabilities also for the 38-atom truncated octahedron and for the Mackay icosahedron ${ }^{24.25}$, except that the Gupta potential favours the complete 55-atom icosahedron 24 Very different structures were found, however, with the Sutton-Chen ${ }^{29}$ and glue ${ }^{30}$ potentials. The former favours unusual, somewhat disordered structures, that are a hybrid of close packed structures, decahedra and Mackay icosahedra.21.31.32 A still different set of structures were found with the glue model, which favours polytetrahedral structures in the whole size range, except for those sizes close to the complete Mackay icosahedra 22

It is the aim of the present paper to use simulation techniques to study the melting of $\mathrm{Al}$ clusters in the size range studied experimentally,,$\frac{5}{5}$ in particular to see if the experimental trends can be captured by semi-empirical potentials. Besides, reliably finding the global minima and performing long enough simulations to achieve wellconverged thermodynamic results is only tractable for 
such potentials. Several potentials that were fitted to $\mathrm{Al}$ properties have been suggested. 26.27 .28 .29 .30 Of these, we have chosen to use the Gupta ${ }^{28}$ and glue ${ }^{30}$ potentials, as they cover two very different set of structures ${ }^{22.23 .24}$ For sodium clusters, it has now been shown that the size dependence of the melting behaviour primarly reflects the geometric structure of the solid clusters 33 So, it is our hope that the behaviour exhibited by these two potentials will be representative of those that favour icosahedral and polytetrahedral structures, respectively, in this size range. It is noteworthy that high symmetry polytetrahedral structures are possible $\left(D_{6 d}\right.$ at $N=51, D_{3 h}$ at $N=57$, and $T_{d}$ at $\left.N=61\right)^{34.35}$ at the sizes for which the experimental latent heat showed a maximum, making it particularly interesting to study a potential that favours polytetrahedral structures.

\section{METHODS}

We performed canonical Monte Carlo (MC) simulations using the parallel tempering (PT) $\operatorname{method}^{36}$ to study the melting of Al clusters in the size range $N=$ 49 - 62. Our simulations consisted of 10 million MC steps, following an initial equilibration period of 1 million steps, for each of the 48 trajectories used, whose temperatures ranged from 10 to $1000 \mathrm{~K}$ depending on the cluster size and on the model. All the trajectories were initialized with the ground-state structures for each size and model. ${ }^{22,24}$ The lowest energy geometries for sizes not reported previously were obtained using a basin-hopping global optimization method ${ }^{37}$ Exchange among different temperatures was attempted with a probability of $10 \%$. Evaporation or fragmentation of clusters was avoided by adding a spherical repulsive hard wall of radius $r_{0}+r$, where $r$ is the cluster ground-state radius (calculated as the distance from the center of mass to the furthest atom), and $r_{0}$ the equilibrium bulk interatomic distance for Gupta and 3/2 of the dimer bond length for the glue model. These constraining radii were large enough not to affect the location of the main peak in the heat capacity curves. The data from these simulations was processed using the multihistogram technique ${ }^{2}$

The two potentials used belong to the family of embedded-atom potentials. They comprise a pair potential plus a many-body term that depends on the electronic density at the atomic position. Therefore, the total energy can be written:

$$
E=\sum_{i} \sum_{j \neq i} \phi\left(r_{i j}\right)+\sum_{i} F\left(\bar{\rho}_{i}\right),
$$

where $\phi\left(r_{i j}\right)$ is a pair potential, $r_{i j}$ is the interatomic distance between atoms $i$ and $j, F\left(\bar{\rho}_{i}\right)$ is an embedding function, and $\bar{\rho}_{i}$ is the electronic density at site $i$, which is usually approximated as the linear superposition of the atomic densities of the rest of the atoms, i.e. $\bar{\rho}_{i}=\sum_{j \neq i} \rho\left(r_{i j}\right)$. The main difference between the two potentials is that Gupta assumes analytic forms for the
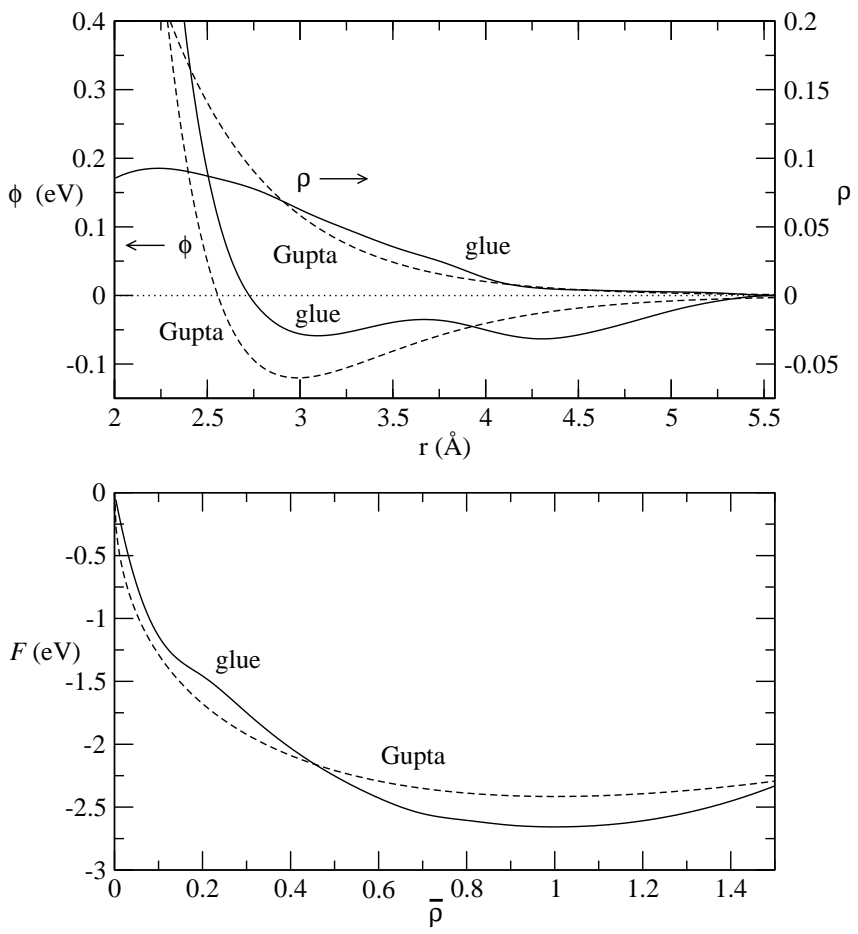

FIG. 1: Comparison of $\phi(r), \rho(r)$ and $F(\bar{\rho})$ for Al Gupta and glue potentials in the effective pair format.

pair potential, the embedding function and the density, 28 whereas, in the glue model, these functions are determined by the fitting process ${ }^{30}$ The best way to compare the two potentials is in the effective pair format 38 The forms for $\phi(r)$ and $F(\bar{\rho})$ are non-unique, and in the effective pair format they are chosen so that the embedding function has a minimum at the value of $\bar{\rho}$ appropriate for the crystal. As can be seen from Fig. 11 there are considerable differences between the two potentials. For example, the pair potential for the glue potential is significantly shallower, and shows a double well structure as compared to the single well in the Gupta pair potential.

The potentials also differ in the number of properties and configurations that have been used in the fitting process. The glue model was adjusted to reproduce the forces obtained by first-principles calculations for a variety of environments, including surfaces, clusters, liquids and crystals, 30 However, the four free parameters in the Gupta potential have been simply fitted to some Al bulk properties, namely, the lattice parameters and elastic moduli. ${ }^{39}$ Therefore, the glue potential is more likely to work well in a variety of situations, and, for example, has been particularly successful in modelling the self-diffusion in $\mathrm{Al}^{40}$

For both potentials some thermodynamic properties of the Al clusters have been studied previously, ${ }^{24.41 .42 .43}$ but the only overlap with the size range considered here are at $N=54-56$ for the Gupta potential. ${ }^{24,41,42}$ 
(a)
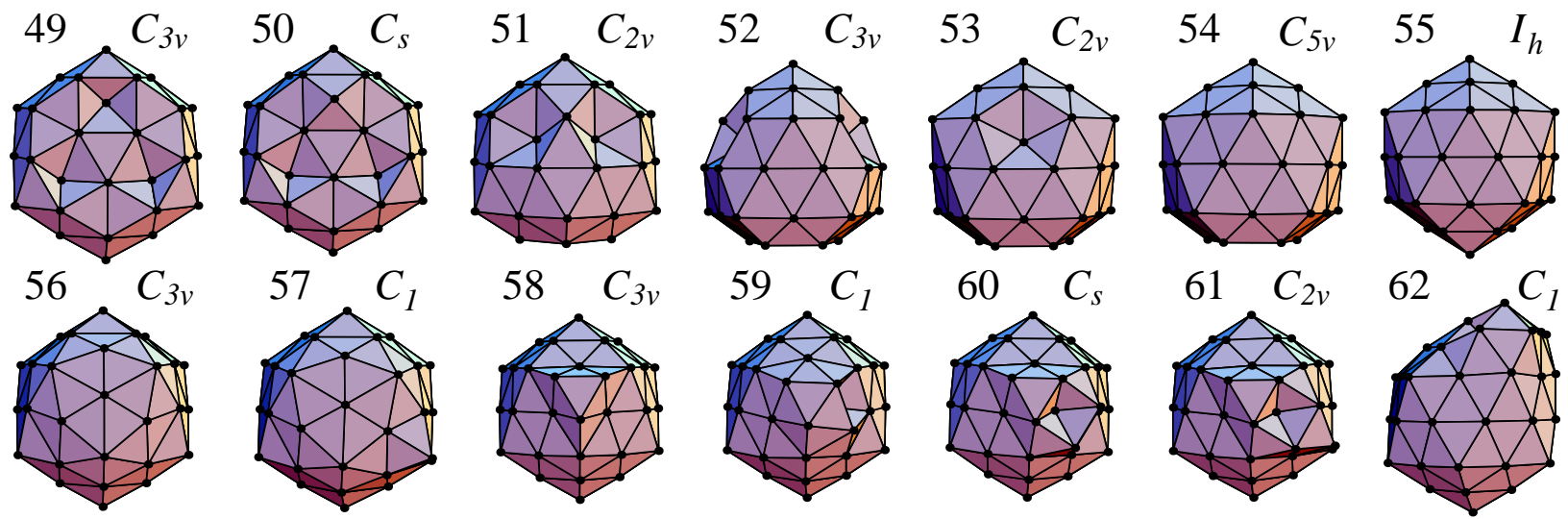

(b)
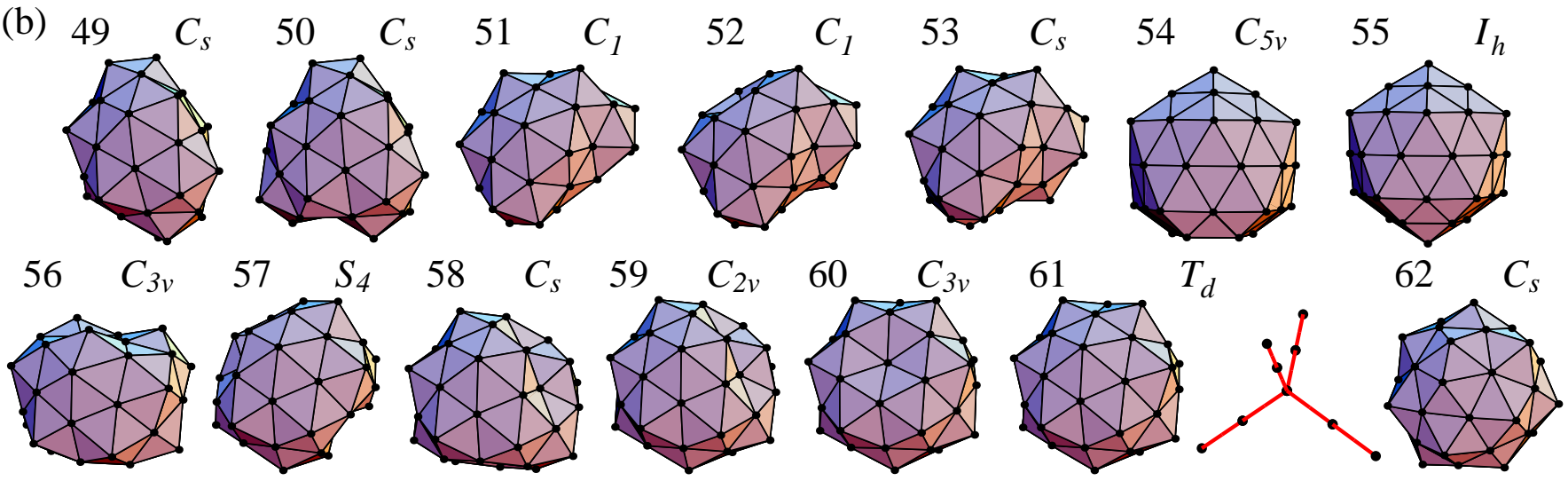

FIG. 2: (Colour online) Ground state structures for Al (a) Gupta and (b) glue clusters with 49 to 62 atoms. For the 61-atom glue cluster, the disclination network is also depicted.

\section{RESULTS}

The structures of the global minima for this size range are depicted in Fig 2] For the Gupta potential, all the structures are based upon the Mackay icosahedron, whereas for the glue potential, they are all polytetrahedral (i.e., the whole structure can be divided up into tetrahedra with atoms at the vertices), except at $N=$ 54 and 55, which are icosahedral. For the Gupta potential, the 55-atom Mackay icosahedron is most stable, whereas for the glue potential, the 55-atom and 61-atom structures are particularly stable. For polytetrahedral structures, it is generally preferred to have five tetrahedra around a nearest-neighbour contact, but beyond a certain size, edges surrounded by six tetrahedra must also be present. The network formed by these sixfold edges, termed disclination network, provides an useful way to characterize polytetrahedral structures. For $N=$ 61 the tetrahedral disclination network is depicted in Fig. 2 and most of the other polytetrahedral structures are based upon this structure. It is noteworthy that the possible high-symmetry polytetrahedral structures at $N=$ 51 and 57, which involve a linear and a trigonal disclination network, mentioned in the introduction as potential candidates for the experimental peaks in the latent heat, are not favoured by this potential. However, whether the 61-atom structure leads to a particularly large latent heat, should provide an indicator of whether this suggestion could be correct.

That the two potentials show completely different structures does not necessarily mean that one or both are bad potentials, but illustrates how difficult it is for a potential to correctly predict a cluster's structure, because to do so, the potential must be able to model a whole host of bulk and surface properties of the material correctly. Indeed, it is not uncommon for potentials that purport to model the same material to exhibit very different structures $\underline{\underline{44}}$

Figure 3 shows the calculated canonical heat capacities as a function of temperature for $\mathrm{Al}$ clusters with 49 to 62 atoms using the Gupta and glue models. The heat capacity curves for the two potentials show few similarities. The Gupta heat capacities show a fairly well-defined peak for almost all sizes, as expected for clusters with icosahedral geometries, and the results for $N=54-56$ are consistent with previous results ${ }^{24.41 .42}$ Interestingly, premelting effects are observed for the sizes $N=58-62$.

The glue potential, instead, predicts a smooth transition from the solid to the liquid state without an associated latent heat or peak in the heat capacity curve. 

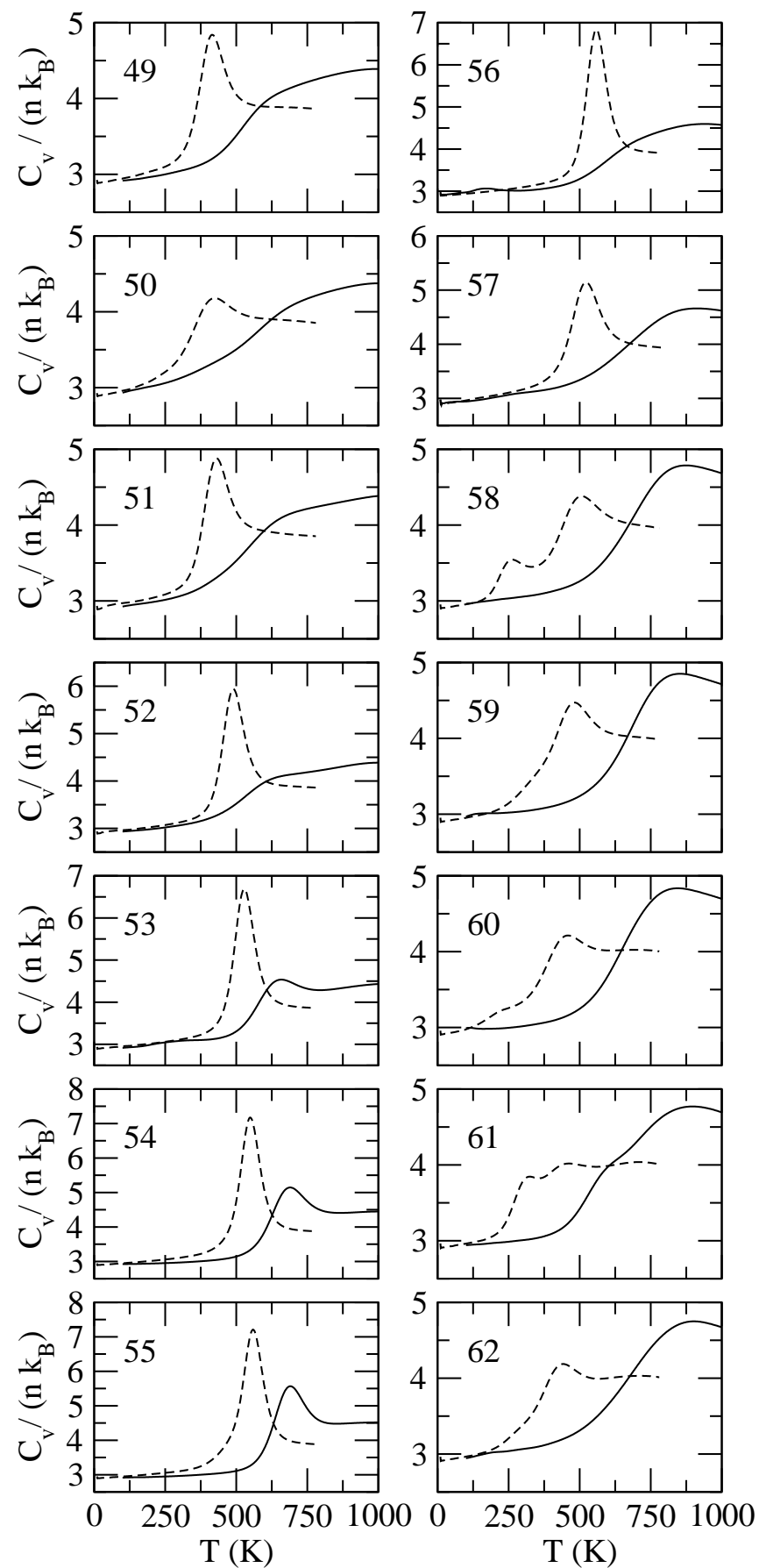

FIG. 3: Heat capacities as a function of temperature for aluminium clusters from 49 to 62 atoms, predicted by the glue (solid line) and Gupta (dashed line) models.

The sizes $N=54$ and 55, which have icosahedral groundstate structures, represent an exception, and their heat capacity curves are more similar to that for the Gupta potential, showing a well-defined peak albeit broader and at higher temperature than for Gupta. Similar behaviour was observed in a previous simulation study using the glue model $\stackrel{43}{\underline{3}}$ Sun and Gong found a well-defined peak

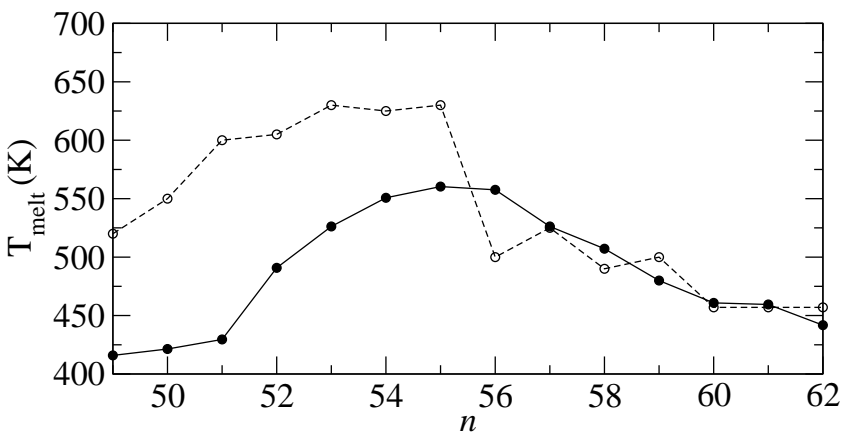

FIG. 4: Comparison of the experimental melting temperatures (open circles) with those predicted by Gupta (closed circles).

in the heat capacity of the icosahedral clusters at $N=$ 13 and 147 , but the polytetrahedral 43-atom cluster underwent a more continuous transition, without a clear peak in the heat capacity. Small peaks are found in the low temperature region of the heat capacity curves of the clusters $\mathrm{Al}_{53}, \mathrm{Al}_{56}$ and $\mathrm{Al}_{57}$, which are indicative of premelting effects.

The fact that the two potentials predict significantly different melting behaviours is not surprising, given the differences in the structure of the lowest-energy clusters. The absence of a well-defined heat capacity peak for clusters with polytetrahedral global minima reflects the basic structural similarities between the ground-state and the molten clusters. It is well-known that simple liquids have substantial polytetrahedral character $\stackrel{45}{ }$ Hence, melting in these clusters is associated with the gradual occupation of structurally similar isomers of higher and higher energy, rather than the more usual cooperative transition between two sets of structures that have different energies and entropies. Similar behaviour is also seen for Lennard-Jones clusters with 25-30 atoms, as they have polytetrahedral global minima $\stackrel{46}{=} \mathrm{A}$ more cooperative, first-order-like transition only appears once the structure of the global minimum changes. Sun and Gong were right to describe such a continuous melting as more akin to the melting of a "glass" (or perhaps more properly an "ideal glass" as the clusters are always in equilibrium) than the melting of an ordered solid that has a fundamentally different 'symmetry' to that of the liquid.

The results obtained by either potential differ significantly from those obtained in the experiments of Breaux et $a l^{\underline{5}}$ Of the two models studied, only the Gupta heat capacities show well-defined peaks for most sizes, as observed in experiments. The continuous nature of the melting transition for most of the glue $\mathrm{Al}$ clusters qualitatively disagrees with experiments. Breaux et al. also reported a non-monotonic variation of the melting temperature with the cluster size, with a maximum at $N=$ 55 and a sharp drop for larger sizes. In Fig. 4 the size dependence of the Gupta and experimental melting temperatures is compared. There is some agreement, but also 


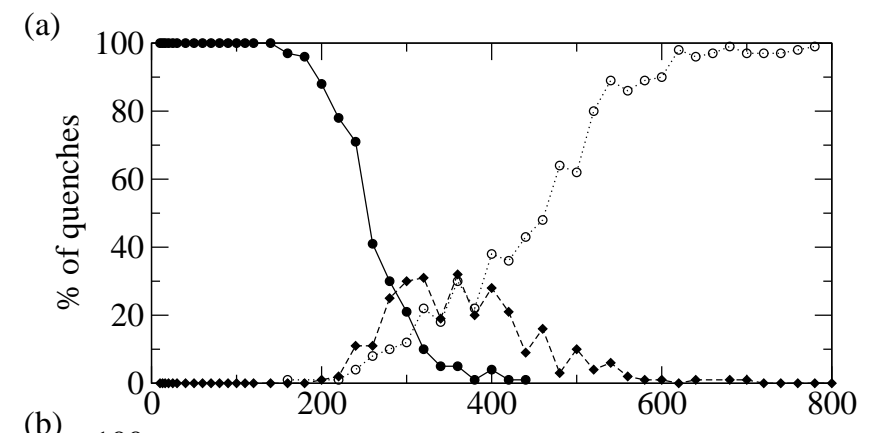

(b)

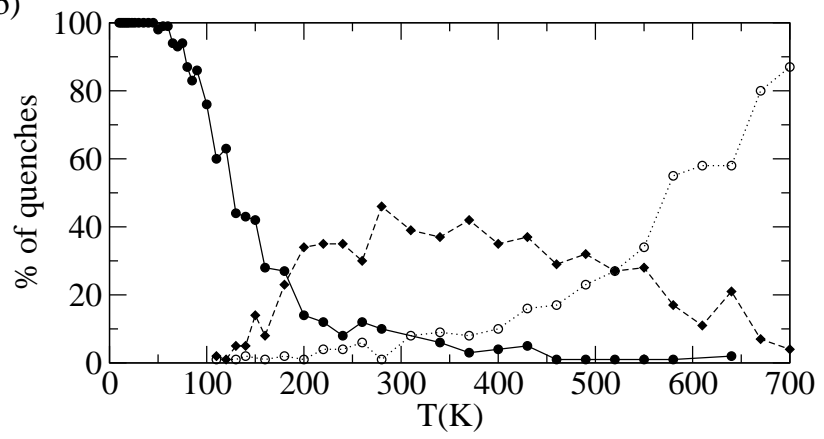

FIG. 5: A structural analysis of the melting of the (a) Gupta $\mathrm{Al}_{58}$ and (b) glue $\mathrm{Al}_{56}$ clusters. The percentage of quenches leading to the icosahedral ground-state structure (closed circles), to structures which have absorbed some or all the extra atoms in the surface (diamonds), and to high energy isomers (open circles) is plotted. In (b) the structures considered are the polytetrahedral ground-state structure (closed circles), an icosahedral isomer (diamonds) and high energy isomers (open circles).

significant differences. As one would expect for clusters with Mackay-based structures, the Gupta clusters exhibit a maximum at the complete 55-Mackay icosahedron magic number, and the melting temperature decreases monotonically on either adding or removing atoms. However, this maximum is broad and there is not the sharp decrease seen experimentally in going from $N=55$ to 56 . This broadness is probably due to compensating changes to both the energy and entropy of melting. ${ }^{47}$ Instead, the latent heat of melting is a more structurally sensitive quantity ${ }^{33}$ The latent heats obtained from the Gupta clusters show a size dependence very similar to the one found for the melting temperature (not shown), and the experimentally observed peaks at $N=51,57$ and 61 are not reproduced by our simulations. As for the sizes at which premelting effects occur, there is disagreement both between our results and the experiments, and between the two models. In particular, the premelting effects at $N=51$ and 52 were not reproduced by either of the models.

Even though experiments concern positively charged clusters and our calculations were performed for neutral clusters, it is unlikely that the discrepancies can be attributed to the charge. The effect that charge might have in such large clusters is probably small, as has been il-

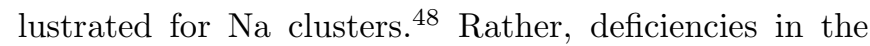
potentials, and hence the structures they predict, are the more likely cause.

In what follows we will analyse the origin of the premelting effects for both potentials. For that purpose, the microscopic behaviour of the system was followed by performing systematic quenches starting from each one of the trajectories. The $\mathrm{Al}_{58}$ Gupta and $\mathrm{Al}_{56}$ glue clusters were chosen as examples that show premelting effects with each potential.

In Fig. 5 we have plotted the percentage of quenches that lead to a particular minimum for Gupta $\mathrm{Al}_{58}$. The ground-state structure of this cluster is a Mackay icosahedron, with the three adatoms located at anti-Mackay positions, all on the same face (Fig. 2). The percentage of quenches that go to this structure is very high at low temperatures, but drops abruptly between 200 and $300 \mathrm{~K}$. Between approximately $250 \mathrm{~K}$ to $500 \mathrm{~K}$, the lowest-energy minimum coexists with a family of isomers in which some or all the extra atoms, that lie above the surface in the ground state, are accommodated in the surface. Note that similar structures consisting of a Mackay icosahedron with one of the extra atoms absorbed in its surface are the ground-state structures for $\mathrm{Al}_{56}$ and $\mathrm{Al}_{57}$ (Fig. 21). At temperatures higher than $550 \mathrm{~K}$, high energy isomers dominate and the cluster is completely melted. Therefore, the first peak in the heat capacity of Gupta $\mathrm{Al}_{58}$ can be attributed to a surface reconstruction in which some or all the extra atoms accommodate in the surface. The same type of surface reconstruction was seen for the other Gupta clusters that showed premelting effects, e.g. for $\mathrm{Al}_{57}$ an isomer in which the two extra atoms are accommodated in the surface becomes competitive above $200 \mathrm{~K}$. Intuitively, these structures will become less favourable as the number of atoms in the third shell of the Mackay icosahedron increases, which is consistent with the fact that premelting features become more subtle for larger sizes.

For all sizes at which the glue model exhibits premelting effects, namely, $N=53,56$ and 57 , the lowest-lying minima are polytetrahedral ${ }^{22}$ However, for $\mathrm{Al}_{56}$ an icosahedral isomer becomes competitive at $180 \mathrm{~K}$, and approximately $50 \%$ of the quenches lead to this minimum up to approximately $580 \mathrm{~K}$ (see Fig. 5). Only above this temperature do high-energy isomers dominate. Similar analyses with analogous results were also performed for $\mathrm{Al}_{53}$ and $\mathrm{Al}_{56}$. From the microscopic analysis it seems clear that the premelting effects observed in the glue model can be attributed to this solid-solid transition from polytetrahedral to icosahedral structures.

Such a transition from polytetrahedral to icosahedral structures is somehow surprising. Even though icosahedral structures are energetically competitive for the nearest sizes to a shell closing, ${ }^{22}$ the solid-solid transition can only be explained if the icosahedral isomers have higher vibrational entropy. However, for pair potentials at least, this is not the usual situation; instead, polytetrahedral structures usually have a higher vibrational entropy be- 
cause of the greater internal strains ${ }^{49}$ The situation is reversed here. For $\mathrm{Al}_{56}$, the geometric mean vibrational frequency is 1.0533 times larger for the polytetrahedral structure, which means that they have a lower vibrational entropy. An estimate of the solid-solid transition can hence be obtained within the harmonic approximation ${ }^{49}$ Following this procedure, the solid-solid transition is predicted to take place at $160 \mathrm{~K}$, which is in very good agreement with the temperature of $168 \mathrm{~K}$ obtained from our PT simulations.

\section{CONCLUSIONS}

We have studied the melting behaviour of $\mathrm{Al}$ clusters in the range $N=49-62$ by means of PT MC simulations using two different models. The results are qualitatively and quantitatively different depending on the potential used, and neither of the potentials is able to reproduce the melting behaviour observed experimentally, ${ }^{\underline{5}}$ Many of the differences in the melting behaviour of the two models can be attributed to the different lowest-energy structures, which are mainly polytetrahedral for the glue potential and predominantly icosahedral for the Gupta potential. These results therefore suggest that neither polytetrahedral or icosahedral structures can explain the experimental size-dependence of the melting behaviour. Instead, the actual structure of $\mathrm{Al}$ clusters in this size range remains a mystery. Furthermore, we feel that none of the other semiempirical potentials are any more likely to be successful in reproducing the $\mathrm{Al}$ thermal properties. An explanation of the experimental results would need, therefore, the application of models at a higher level of theory, but the computational demands of those techniques are significantly higher.

Nevertheless, the results shown here have helped to understand what kind of melting behaviour can be expected from potentials with different structural preferences. The melting transition for potentials that favour polytetrahedral structures is more akin to an ideal glass transition than the finite size equivalent of the bulk first-order melting transition. It is particularly interesting that this more continuous character of the melting transition even holds for sizes, such as $N=61$, at which the polytetrahedral global minimum is particularly stable. Also, due to the larger relative importance of the many-body term over the pair potential, unusual features, such as the solid-solid transition from polytetrahedral to icosahedral structure driven by the vibrational entropy, can be found. However, models that exhibit icosahedral structures will tend to exhibit well-defined heat capacity peaks akin to bulk melting.

\section{Acknowledgments}

The authors are grateful to the Ramón Areces Foundation (E.G.N.) and the Royal Society (J.P.K.D.) for financial support.
1 R. S. Berry, J. Jellinek, and G. Natanson, Chem. Phys. Lett. 107, 227 (1984).

${ }^{2}$ P. Labastie and R. L. Whetten, Phys. Rev. Lett. 65, 1567 (1990).

3 A. A. Shvartsburg and M. F. Jarrold, Phys. Rev. Lett. 85, 2530 (2000).

${ }^{4}$ G. A. Breaux, R. C. Benirschke, T. Sugai, B. S. Kinnear, and M. F. Jarrold, Phys. Rev. Lett. 91, 215508 (2003).

${ }^{5}$ G. A. Breaux, C. M. Neal, B. Cao, and M. F. Jarrold, Phys. Rev. Lett. 94, 173401 (2005).

${ }^{6}$ M. Pellarin, B. Baguenard, M. Broyer, J. Lermé, and J. L. Vialle, J. Chem. Phys. 98, 944 (1993).

7 T. P. Martin, U. Näher, and H. Schaber, Chem. Phys. Lett. 199, 470 (1992).

8 H.-P. Cheng, R. S. Berry, and R. L. Whetten, Phys. Rev. B 43, 10647 (1991).

9 J.-Y. Yi, D. J. Oh, and J. Bernholc, Phys. Rev. Lett. 67, 1549 (1991).

10 R. O. Jones, Phys. Rev. Lett. 67, 224 (1991).

11 S. H. Yang, D. A. Drabold, J. B. Adams, and A. Sachdev, Phys. Rev. B 47, 1567 (1993).

12 J. Akola, M. Manninen, H. Häkkinen, U. Landman, X. Li, and L.-S. Wang, Phys. Rev. B 60, R11297 (1999).

13 R. Ahlrichs and S. D. Elliott, Phys. Chem. Chem. Phys. 1, 13 (1999).

14 S. Valkealahti and M. Manninen, Phys. Rev. B 50, 17564 (1994).
15 S. Valkealahti, U. Näher, and M. Manninen, Phys. Rev. B 51, 11039 (1995).

16 S. Valkealahti and M. Manninen, Phys. Rev. B 57, 15533 (1998).

17 B. K. Rao and P. Jena, J. Chem. Phys. 111, 1890 (1999).

18 R. L. Johnston and J.-Y. Fang, J. Chem. Phys. 97, 7809 (1992).

19 L. D. Lloyd and R. L. Johnston, Chem. Phys. 236, 107 (1998).

20 L. D. Lloyd, R. L. Johnston, C. Roberts, and T. V. Mortimer-Jones, ChemPhysChem 3, 408 (2002).

21 J. Joswig and M. Springborg, Phys. Rev. B 68, 085408 (2003).

22 J. P. K. Doye, J. Chem. Phys. 119, 1136 (2003).

23 G. W. Turner, R. L. Johnston, and N. T. Wilson, J. Chem. Phys. 112, 4773 (2000).

${ }^{24}$ S. K. Lai, W. D. Lin, K. L. Wu, W. H. Li, and K. C. Lee, J. Chem. Phys. 121, 1487 (2004).

25 A. Sebetci and Z. B. Güvenç, Modelling Simul. Matter. Sci. Eng. 13, 683 (2005).

${ }^{26}$ H. Cox, R. L. Johnston, and J. N. Murrell, Surf. Sci. 373, 67 (1997).

27 A. F. Voter and S. P. Chen, Mater. Res. Soc. Symp. Proc. 82, 175 (1987).

28 R. P. Gupta, Phys. Rev. B 23, 6265 (1981).

29 A. P. Sutton and J. Chen, Philos. Mag. Lett. 61, 139 (1990). 
${ }^{30}$ F. Ercolessi and J. B. Adams, Europhys. Lett. 26, 583 (1994).

31 J. P. K. Doye, unpublished.

32 As the $n / m$ value for the Al Sutton-Chen potential $(7 / 6)$ is close to one, in the effective pair format, there is only a shallow well in the pair potential and so the observed structures are somewhat similar to those seen for $\mathrm{Zn}$ and Cd Gupta potentials, which also have very shallow wells in their effective pair potentials ${ }^{50}$.

${ }^{33}$ H. Haberland, T. Hippler, J. Donges, O. Kostko, M. Schmidt, and B. von Issendorff, Phys. Rev. Lett. 94, 035701 (2005).

34 J. P. K. Doye and D. J. Wales, J. Chem. Soc., Faraday Trans. 93, 4233 (1997).

35 J. P. K. Doye and L. Meyer, Phys. Rev. Lett. 95, 063401 (2005).

36 D. Frenkel and B. Smit, Understanding Molecular Simulation (Academic Press, New York, 2002).

37 D. J. Wales and J. P. K. Doye, J. Phys. Chem. A 101, 5111 (1997).
${ }^{38}$ R. A. Johnson and D. J. Oh, J. Mater. Res. 4, 1195 (1989).

39 F. Cleri and V. Rosato, Phys. Rev. B 48, 22 (1993).

40 N. Sandberg, B. Magyari-Köpe, and T. R. Mattsson, Phys. Rev. Lett. 89, 065901 (2002).

41 J. Jellinek and A. Goldberg, J. Chem. Phys. 113, 2570 (2000).

42 R. Werner, Eur. Phys. J. B 43, 47 (2005).

43 D. Y. Sun and X. G. Gong, Phys. Rev. B 57, 4730 (1998).

44 J. P. K. Doye, Comp. Mater. Sci. in press.

45 D. R. Nelson and F. Spaepen, Solid State Phys. 42, 1 (1989).

46 D. D. Frantz, J. Chem. Phys. 115, 6136 (2001).

47 M. Schmidt, J. Donges, T. Hippler, and H. Haberland, Phys. Rev. Lett. 90, 103401 (2003).

${ }^{48}$ F. Calvo and F. Spiegelmann, J. Chem. Phys. 112, 2888 (2000).

49 J. P. K. Doye and F. Calvo, J. Chem. Phys. 116, 8307 (2002).

50 J. P. K. Doye, Phys. Rev. B 68, 195418 (2003). 